\title{
Effect of Flooding and the nos $Z$ Gene in Bradyrhizobia on Bradyrhizobial Community Structure in the Soil
}

 \\ REIKO SAMESHIMA-SAITO ${ }^{3}$, MANABU ITAKURA ${ }^{4,5}$, KIWAMU MINAMISAWA ${ }^{4}$, and AKIHIRO YAMAMOTO ${ }^{1}$ \\ ${ }^{1}$ Faculty of Agriculture, University of Miyazaki, Miyazaki 889-2192, Japan; ${ }^{2}$ Faculty of Life and Environmental Science, Shimane \\ University, Shimane 690-8504, Japan; ${ }^{3}$ College of Agriculture, Academic Institute, Shizuoka University, Shizuoka 422-8529, \\ Japan; ${ }^{4}$ Graduate School of Life Sciences, Tohoku University, Sendai, Miyagi 980-8577, Japan; ${ }^{5}$ Center for Ecological \\ Evolutionary Developmental Biology, Kyoto Sangyo University, Kyoto 603-8555, Japan; and ${ }^{6}$ College of Agriculture, Central \\ Luzon State University, Science City of Muñoz, 3120 Nueva Ecija, Philippines
}

(Received September 1, 2016-Accepted April 11, 2017-Published online June 8, 2017)

We investigated the effects of the water status (flooded or non-flooded) and presence of the nos $Z$ gene in bradyrhizobia on the bradyrhizobial community structure in a factorial experiment that examined three temperature levels $\left(20^{\circ} \mathrm{C}, 25^{\circ} \mathrm{C}\right.$, and $30^{\circ} \mathrm{C}$ ) and two soil types (andosol and gray lowland soil) using microcosm incubations. All microcosms were inoculated with Bradyrhizobium japonicum USDA6 ${ }^{\mathrm{T}}$, B. japonicum USDA123, and B. elkanii USDA76 ${ }^{\mathrm{T}}$, which do not possess the nosZ gene, and then half received $B$. diazoefficiens USDA $110^{\mathrm{T}} \mathrm{wt}$ (wt for the wild-type) and the other half received $B$. diazoefficiens USDA110 $\Delta$ nos $Z$. USDA $110^{\mathrm{T}} \mathrm{wt}$ possesses the nos $Z$ gene, which encodes $\mathrm{N}_{2} \mathrm{O}$ reductase; $110 \Delta$ nos $Z$, a mutant variant, does not. Changes in the community structure after 30- and 60-d incubations were investigated by denaturing-gradient gel electrophoresis and an image analysis. USDA $6^{\mathrm{T}}$ and $76^{\mathrm{T}}$ strains slightly increased in non-flooded soil regardless of which USDA110 ${ }^{\mathrm{T}}$ strain was present. In flooded microcosms with the USDA $110^{\mathrm{T}} \mathrm{wt}$ strain, USDA $110^{\mathrm{T}} \mathrm{wt}$ became dominant, whereas in microcosms with the USDA110 $\operatorname{nos} Z$, a similar change in the community structure occurred to that in non-flooded microcosms. These results suggest that possession of the nos $Z$ gene confers a competitive advantage to $B$. diazoefficiens USDA110 ${ }^{\mathrm{T}}$ in flooded soil. We herein demonstrated that the dominance of $B$. diazoefficiens USDA $110^{\mathrm{T}} \mathrm{wt}$ within the soil bradyrhizobial population may be enhanced by periods of flooding or waterlogging systems such as paddy-soybean rotations because it appears to have the ability to thrive in moderately anaerobic soil.

Key words: Bradyrhizobium, community structure, denitrification, nos Z, flooded condition

The genus Bradyrhizobium is a group of soil bacteria that is active in symbiotic nitrogen fixation with soybeans and other leguminous plants. The nitrogen fixing ability of soybean bradyrhizobia depends on the strains present and environmental conditions involved in symbiosis $(16,32)$. The major nodulating rhizobia in soybean (Glycine max. [L.] Merr.) are Bradyrhizobium japonicum, B. elkanii, and Sinorhizobium (Ensifer) fredii (4, 14, 15, 17, 31, 45). The novel species, $B$. diazoefficiens, has been proposed as an independent species from B. japonicum (6), and B. diazoefficiens USDA $110^{\mathrm{T}}$ is referred to in this study rather than B. japonicum USDA110. Although other species of rhizobia have been implicated in soybean nodulation, the major endosymbionts in Japan are considered to be B. japonicum, B. diazoefficiens, and $B$. elkanii in acidic-neutral soils and $S$. fredii in alkaline soils $(20,23,25,26,30,39)$. Soybean-nodulating bacteria are widely distributed throughout the world, and endemism in their genetic diversity reflects the geographical and climatic differences as well as diversity of local soybean varieties. Therefore, analyses on the genetic diversity and distributions of indigenous soybean-nodulating rhizobia are important for improving our understanding of rhizobial ecology under various environmental conditions. Furthermore, an understanding of the functional relationships between different genetic types

\footnotetext{
* Corresponding author. E-mail: yt-saeki@cc.miyazaki-u.ac.jp;
} Tel: +81-985-58-7206; Fax: +81-985-58-7206. and environmental conditions will contribute to the more informed management of soybean production.

Denitrification ability, anaerobic respiration that reduces nitrate to nitrogen gas, also varies among strains of bradyrhizobia $(28,38)$. Some strains display complete denitrification activity and are able to release dinitrogen gas. Others show incomplete denitrification, which releases nitrous acid $\left(\mathrm{NO}_{2}^{-}\right)$, nitric oxide $(\mathrm{NO})$, or nitrous oxide $\left(\mathrm{N}_{2} \mathrm{O}\right)$. Some strains appear to show no denitrification activity. Denitrification by soil bacteria generally requires four enzymes: periplasmic nitrate reductase (Nap), nitrite reductase (Nir), NO reductase (Nor), and $\mathrm{N}_{2} \mathrm{O}$ reductase (Nos). The respective genes that encode these enzymes are nap $A$, nirK, $\operatorname{nor} C B$, and $\operatorname{nos} Z(2,37)$. Nitrous oxide is an important greenhouse gas with a $100-\mathrm{yr}$ global warming potential of $298 \mathrm{CO}_{2}$ equivalents (12). Therefore, the mitigation of $\mathrm{N}_{2} \mathrm{O}$ release from the soybean rhizosphere, which is dominated by strains that display incomplete denitrification, is an important research task for sustainable soybean production $(10,11)$.

Bradyrhizobium diazoefficiens strain USDA $110^{\mathrm{T}}$ is known to have high nitrogen fixation activity, and is regarded as a useful rhizobial strain capable of inducing high soybean yield $(9,37)$. In addition, strain USDA $110^{\mathrm{T}}$ displays complete denitrification activity and may contribute to reducing the emission of $\mathrm{N}_{2} \mathrm{O}(13,29)$. Therefore, USDA $110^{\mathrm{T}}$, with its high nitrogen fixation and complete denitrification activities, is expected to become an important rhizobial strain for sus- 
tainable soybean production. A thorough understanding of the physiological ecology of bradyrhizobia is important for ensuring the infection of soybean crops with useful Bradyrhizobium strains such as B. diazoefficiens USDA110 .

Our previous studies $(23,25-27)$ demonstrated that ecological niches for the bradyrhizobial community structure are arranged along a latitude gradient, suggesting that temperature is one of the important environmental factors in bradyrhizobial ecology. Furthermore, an analysis of the community structure of indigenous rhizobia showed that strains belonging to the Bj110 cluster, which is the cluster of USDA $110^{\mathrm{T}}$ based on a diversity analysis of the $16 \mathrm{~S}-23 \mathrm{~S}$ rRNA gene internal transcribed spacer (ITS) region, are abundant in silty soils such as fields converted from paddy rice production (26). Shiina et al. (33) also demonstrated that strains belonging to the cluster of USDA $6^{\mathrm{T}}$ lacking the $n o s Z$ gene were dominant in andosols, whereas those belonging to the cluster of USDA $110^{\mathrm{T}}$ possessing the nosZ gene were dominant in alluvial soils. These findings suggest that the endemism of bradyrhizobia depends on certain soil properties, and, thus, may affect denitrification activity through the presence or absence of strains that exhibit $\mathrm{N}_{2} \mathrm{O}$ reductase activity. Strains classified into those belonging to the Bj110 cluster possessing the nos $Z$ gene may also be able to survive well in anaerobic soils with low redox potential, as typically occurs in paddy fields.

Based on previous findings $(26,33)$ showing that strains possessing the nos $Z$ gene are abundant in fields converted from paddy rice fields, we hypothesize that the reducing conditions caused by waterlogging or flooding will be advantageous for strains that exhibit complete denitrification activity rather than those that are incapable of or only capable of incomplete denitrification. In order to test this hypothesis, we investigated the effects of water flooding on the community structure of bradyrhizobia at different temperatures and oxidation-reduction conditions in two different soils using microcosm incubations. Specifically, we compared differences in changes in the community structure over time with and without flooding in different soils with the USDA $110^{\mathrm{T}}$ strain possessing the nos $Z$ gene or a mutant variant lacking the $\operatorname{nos} Z$ gene.

\section{Materials and Methods}

The experiment was conducted with controlled microcosm incubations in a full factorial design involving two soil types (andosol and gray lowland soil), two flooding conditions (flooded or nonflooded), three temperature conditions (high, medium, and low), and two kinds of bradyrhizobial communities (the substitution of one out of four strains between two variants of the strain, one with the nos $Z$ gene and one without). The response variable was the relative abundance of the various strains of added bradyrhizobia after a period of incubation. The community structure was assayed at 30 and $60 \mathrm{~d}$, giving two incubation periods. All unique treatment level combinations were replicated three times. Details of the methods are stated henceforth.

\section{Soils and bradyrhizobia}

Two different soils, an andosol (pH 6.28, EC $0.038 \mathrm{dS} \mathrm{m}^{-1}$, T-C: $4.63 \%$, T-N: $0.34 \%, \mathrm{C} / \mathrm{N}$ ratio: 13.6$)$ and gray lowland soil $(\mathrm{pH}$ 6.05, EC $0.102 \mathrm{dS} \mathrm{m}{ }^{-1}$, T-C: $1.75 \%$, T-N: $0.19 \%$, C/N ratio: 9.4 ), were collected from the experimental fields of the University of Miyazaki, Japan. The soil groups to which the soils belonged were identified by the Classification of cultivated soils in Japan, Third approximation (5). Air-dried soil was passed through a sieve with a 2-mm screen, then sterilized by autoclaving three times at $121^{\circ} \mathrm{C}$ for 20 min with a 1-d interval between the second and third autoclave treatments. In each incubation, four Bradyrhizobium strains were used to create a bradyrhizobial community representative of those found in Japan (26). They were as follows: 1) B. japonicum strain USDA $6{ }^{\mathrm{T}}$, which releases $\mathrm{N}_{2} \mathrm{O}$ as the end product of denitrification, 2) B. japonicum strain USDA123, which lacks any denitrification activity, 3) $B$. elkanii strain USDA76 ${ }^{\mathrm{T}}$, which releases $\mathrm{NO}_{2}{ }^{-}$as the end product of denitrification, and 4) $B$. diazoefficiens strain USDA $110^{\mathrm{T}}$ wt (wild-type), which releases $\mathrm{N}_{2}$ by complete denitrification (28), or B. diazoefficiens USDA110 1 nosZ, which is a mutant variant of USDA $110^{\mathrm{T}}$ that lacks an active $\mathrm{N}_{2} \mathrm{O}$ reductase gene (8).

\section{Preparation and incubation of microcosms}

Each microbial strain stored on YMA slant medium (41) at $4{ }^{\circ} \mathrm{C}$ used in the experiment was cultured for $5 \mathrm{~d}$ in $10 \mathrm{~mL}$ of YMB medium (41) at $145 \mathrm{rpm}$ in a Multi Shaker (EYELA MMS-210, Tokyo Rikakikai, Tokyo, Japan) at $28^{\circ} \mathrm{C}$ in the dark. The cell density of each cultured strain was measured under a microscope by means of a hemocytometer for bacteria (Erma Inc., Tokyo, Japan) to achieve approximately $10^{5}$ cells $\mathrm{g}^{-1}$ (dry soil basis) for each strain, and each strain was mixed with prepared soil as a set of WT strains (WT set: USDA6 $6^{\mathrm{T}}, 76^{\mathrm{T}}, 110^{\mathrm{T}}$, and 123) or a set of a mutant and WT strains (Mut set: USDA6 ${ }^{\mathrm{T}}, 76^{\mathrm{T}}, 110 \Delta$ nosZ, and 123). In the preparation of microcosms, USDA6 ${ }^{\mathrm{T}}$ was set as dominant for andosol and USDA $110^{\mathrm{T}}$ was set as dominant for gray lowland soil according to the previous study by Shiina et al. (33) that demonstrated the dominance of each strain depending on the soil type. The soil water content was adjusted to $40 \%$ of the maximum water-holding capacity with sterile distilled water. Potassium nitrate was added to microcosm soils at a rate of $50 \mathrm{mg} \mathrm{NO}-\mathrm{N} \mathrm{kg}^{-1}$ dry soil. Microcosms were created by placing aliquots of $20 \mathrm{~g}$ (dry soil basis) of prepared soil into 50-mL sterile conical tubes with a gas-permeable filter cap (CELLSTAR, Cellreactor tube $50 \mathrm{~mL}$ with a Blue filter screw cap, Greiner Bio-One, Tokyo, Japan). Microcosms containing andosol or gray lowland soil were termed ADS or GLS microcosms, respectively. In non-flooded microcosms, soil was maintained at $40 \%$ water content of the maximum water-holding capacity. In flooded microcosms, soil was kept saturated with sterile distilled water with a free water content at $5 \mathrm{~cm}$ above the level of the soil surface. The three levels of incubation temperatures were $20^{\circ} \mathrm{C}$ (low), $25^{\circ} \mathrm{C}$ (medium), and $30^{\circ} \mathrm{C}$ (high) based on mean temperatures during rice paddy production in northern and southern regions of Japan. The opening of each microcosm was covered with a cap fit with a gas-permeable filter and incubations were performed in the dark in a temperature controlled incubator (TG-180-3LS, NK system, Nippon Medical \& Chemical Instruments, Osaka, Japan). Microcosms were weighed and supplied with sterile distilled water every 3-4 d in nonflooded microcosms and every week in flooded microcosms to maintain the soil water condition. The oxidation-reduction potential (ORP) and dissolved oxygen content (DOC) were measured for representative microcosms of each treatment at intervals using a handheld multi-parameter meter (Intelligent Water Checker IWC-5, CUSTOM Corp., Tokyo, Japan) equipped with an ORP sensor (ORP-14 model, CUSTOM Corp) and handheld DO meter (Digital Oxygen DO-5509 model, SATO SHOUJI, Kawasaki, Japan). The ORP and DOC of non-flooded microcosms were assessed in 30-and $60-d$ incubation samples by the measurement of soil suspension (soil:water=1:4).

\section{Analysis of bradyrhizobial community structures}

Soil samples of microcosms were sampled immediately after the preparation of microcosms $(0 \mathrm{~d})$ and after 30 - and $60-\mathrm{d}$ incubations, and stored at $20^{\circ} \mathrm{C}$ for later analyses. Environmental DNA (eDNA) was extracted from soil samples using an ISOIL DNA extraction kit (Nippon Gene, Tokyo, Japan) with the following modifications to the manufacturer's procedure: Each sample was pre-treated with a 
bead beater homogenizer (Disruptor Genie, Scientific Industries, Bohemia, NY, USA) by mixing a 0.5 -g soil sample with $950 \mu \mathrm{L}$ of lysis solution HE (Nippon Gene), $50 \mu \mathrm{L}$ of lysis solution $20 \mathrm{~S}$ (Nippon Gene), and $0.5 \mathrm{~g}$ of glass beads $(0.1 \mathrm{~mm}$ in diameter) for approximately $1 \mathrm{~min}$. The following procedures were then conducted in accordance with the manufacturer's instructions. The eDNA extract was purified using a polyvinylpolypyrrolidone spin-column (24). Polymerase chain reaction (PCR) and denaturing-gradient gel electrophoresis (DGGE) analyses were performed according to the methods described by Saeki et al. (24). PCR amplification targeting a part of the 16S-23S rRNA gene ITS region was conducted with Ex-Taq DNA polymerase (Takara Bio, Otsu, Japan), and a primer set for DGGE (DG-ITS-F: 5'-GTCCGCGAAACATCACTT-3', DG-ITS-R-GC clump: 5'-CGCCCGCCGCGCCCCGCGCCCGTC CCGCCGCCCCCGCCCGGTCCACACACTCGGCAGA-3') with extracted eDNA as the template. Touchdown PCR was performed as follows: pre-run at $94^{\circ} \mathrm{C}$ for $5 \mathrm{~min} ; 30$ cycles of denaturation at $94^{\circ} \mathrm{C}$ for $30 \mathrm{~s}$, annealing at $68^{\circ} \mathrm{C}$ in the first cycle, which was lowered by $1^{\circ} \mathrm{C}$ per cycle through the tenth cycle, and at $58^{\circ} \mathrm{C}$ for the remaining 20 cycles for $30 \mathrm{~s}$, then extension at $72^{\circ} \mathrm{C}$ for $30 \mathrm{~s}$; and a final postrun at $72^{\circ} \mathrm{C}$ for $10 \mathrm{~min}$. PCR amplicons were checked by $1.5 \%$ agarose gel electrophoresis in TAE buffer. As a negative control, we confirmed that no PCR amplicon was obtained from non-inoculated samples of the sterilized experimental soils. PCR amplicons were separated on an $8 \%$ polyacrylamide $(2.6 \%$ C) gel with a $41-44 \%$ denaturing gradient at $60^{\circ} \mathrm{C}$ and $100 \mathrm{~V}$ for $14 \mathrm{~h}$ using the D-Code system (BioRad Laboratories, Hercules, CA, USA). After electrophoresis, the relative intensities of the visualized bands were measured using ImageJ program ver.1.48 (http://imagej.nih.gov/ij/) and their relative abundances were calculated from the peak area of each strain.

The abundance of each strain was obtained from the analysis of three replicates. A coded nomenclature was devised to describe each unique treatment combination. The first letter of the code was for soil: A for ADS microcosms or G for GLS microcosms. The second letter represented the water status: $\mathrm{F}$ for flooded or $\mathrm{N}$ for non-flooded. The third letter represented the alternate bradyrhizobia strains: W for microcosms containing the WT set with USDA $110^{\mathrm{T}} \mathrm{wt}$ or $\mathrm{D}$ for microcosms containing the Mut set with USDA110 $\operatorname{nos} Z$. The fourth letter represented the incubation temperature: $\mathrm{H}$ for $30^{\circ} \mathrm{C}, \mathrm{M}$ for $25^{\circ} \mathrm{C}$, and $\mathrm{L}$ for $20^{\circ} \mathrm{C}$. The incubation period was designated by appending 30 for $30 \mathrm{~d}$ and 60 for $60 \mathrm{~d}$.

\section{Estimation of Community Structures}

In order to evaluate the relative position among the community structure, we used the multi-dimensional scaling (MDS) method based on the Bray-Curtis similarity index $(3,19)$. We then used a cluster analysis to group the positions of the community structures based on the results of MDS. The Bray-Curtis similarity index is regarded as a robust index for reflecting the degree of similarities among communities (7). The Bray-Curtis similarity indices $(B C)$ of all pair-wise comparisons were calculated using the following equation:

$B C_{\mathrm{AB}}=\Sigma\left|n_{\mathrm{A}}-n_{\mathrm{B}}\right| /\left(N_{\mathrm{A}}+N_{\mathrm{B}}\right)$,

where $B C_{\mathrm{AB}}$ is the dissimilarity between communities $\mathrm{A}$ and $\mathrm{B}, n_{\mathrm{A}}$ and $n_{\mathrm{B}}$ represent the abundances of a given strain in communities $\mathrm{A}$ and $\mathrm{B}$, respectively, and $N_{\mathrm{A}}$ and $N_{\mathrm{B}}$ represent the total abundance of all strains in communities $\mathrm{A}$ and $\mathrm{B}$, respectively. MDS and cluster analyses were conducted using R software v3.2.1 (http:// www.R-project.org/). The dendrogram for the cluster analysis was constructed by the Ward method, also using R. In addition, to evaluate the relationship between the abundances of the different bradyrhizobia and estimate environmental factors affecting the community structure, we used $\mathrm{R}$ to perform a principal-component analysis (PCA) on the abundance of the results of the microcosms in two separate analyses: one for microcosms with the WT set containing strains USDA $6^{\mathrm{T}}, 76^{\mathrm{T}}, 110^{\mathrm{T}} \mathrm{wt}$, and 123 , and the other for microcosms with the Mut set containing strains USDA6 $6^{\mathrm{T}}, 76^{\mathrm{T}}, 110 \Delta n o s Z$, and 123.

\section{Statistical analysis}

In the estimation of similarities among community structures in microcosms, statistical analyses were conducted using $\mathrm{R}$ software. In the comparison of two levels of treatments such as flooding conditions, Welch's t-test was performed for each strain. In the comparison of three levels of treatments such as temperatures and incubation periods, Bonferroni's significance test was performed for each strain. The effects of incubation factors, the water status, incubation temperatures, and incubation periods on the community structure were estimated with a multivariate analysis of variance (MANOVA) using Pillai's trace statistics.

\section{Results}

\section{Soil ORP and dissolved oxygen over time}

The soil ORP and DOC values of all non-flooded microcosms of andosol and gray lowland soil in 30- and 60-d soil samples indicated oxidative conditions such as $212 \pm 6.2 \mathrm{mV}$ and $7.0 \pm 0.5 \mathrm{mg} \mathrm{L}^{-1}$, and $217 \pm 4.4 \mathrm{mV}$ and $7.1 \pm 0.3 \mathrm{mg} \mathrm{L}^{-1}$ under the measurement conditions, respectively. On the other hand, all flooded microcosms initially had soil ORP values of approximately $200 \mathrm{mV}$, which then declined as the incubation time progressed before reaching equilibrium (Fig. 1). ORP values in GLS microcosms declined more rapidly and were approximately $100 \mathrm{mV}$ at $14 \mathrm{~d}$ and stabilized at approximately $-200 \mathrm{mV}$ at $28 \mathrm{~d}$. In ADS microcosms, ORP values were approximately $+50 \mathrm{mV}$ at $14 \mathrm{~d}$ and then stabilized at approximately -50 to $-100 \mathrm{mV}$ at $60 \mathrm{~d}$. These changes over the course of the incubation period were similar to those for microcosms containing the WT set and Mut set. Thus, the only differences in ORP values during the incubation period were between ADS and GLS microcosms under flooded conditions. The DOC in flooded microcosm soils was similar among soil types and strains, at approximately $1 \mathrm{mg} \mathrm{L}^{-1}$ after



Fig. 1. Oxidation-reduction potential, ORP (A and B) and dissolved oxygen content, DOC (C and D) in flooded microcosms incubated with each of two soil types (andosol and grey lowland soil) at each of three temperature conditions (low $20^{\circ} \mathrm{C}$; medium $25^{\circ} \mathrm{C}$, and high $30^{\circ} \mathrm{C}$ ).

Separate plots are shown for microcosms in which the terms WT set and Mut set contain strain sets, $\mathrm{USDA}^{\mathrm{T}}, 76^{\mathrm{T}}, 110^{\mathrm{T}} \mathrm{wt}$, and 123 (A and C), or $\mathrm{USDA}^{\mathrm{T}}, 76^{\mathrm{T}}, 110 \Delta$ nos $Z$, and 123 (B and D), respectively. Continuous significant differences in ORP values were detected between soil types from 14-d incubations (Welch's t-test, $n=3, p<0.01$ ). 
a 30-d incubation and reaching stable values of approximately $0.3-0.9 \mathrm{mg} \mathrm{L}^{-1}$ from then until the end of the incubation period (Fig. 1). As supplementary data, $\mathrm{pH}$ and EC values were indicated in Table S1. Briefly, EC values decreased under flooded conditions in both soil types, suggesting a decrease in nitrate concentrations. On the other hand, $\mathrm{pH}$ values increased under flooded conditions.

\section{DGGE analysis of bradyrhizobial community structures}

The population density of bradyrhizobia reached approximately $10^{7}-10^{8}$ cells $\mathrm{g}^{-1}$ and became stable during the incuba- tion period (Table S1). The DGGE analysis detected the four Bradyrhizobium strains as separate electrophoresed bands, and the strength of each band was regarded as an index of the abundance of each strain in the soil. The results of the DGGE analysis indicated significant changes in bradyrhizobial community structures during the incubation period in flooded and non-flooded microcosms (Fig. 2 and Table S2). In ADS microcosms with the WT set, very few changes in the relative abundance of the strains occurred in non-flooded microcosms, whereas the abundance of USDA $110^{\mathrm{T}} \mathrm{wt}$ increased significantly at all temperatures in flooded microcosms (Fig.



Fig. 2. The relative abundance of each of four strains comprising the bradyrhizobial community structure in microcosms in an experiment examining combinations of two soil types (A: ADS and B: GLS), two soil moisture conditions (non-flooded, N, and flooded, F), and three temperature conditions (H: $30^{\circ} \mathrm{C}, \mathrm{M}: 25^{\circ} \mathrm{C}, \mathrm{L}: 20^{\circ} \mathrm{C}$ ) after 0-, 30-, and 60-d incubations.

Each value is the mean \pm standard deviation of three replicate microcosms $(n=3)$. A significance test was conducted between incubation periods per strain per microcosm (Bonferroni, $p<0.05$ ). The four strains of Bradyrhizobium were B. japonicum USDA6 ${ }^{\mathrm{T}}$ (U6), B. elkanii USDA76 ${ }^{\mathrm{T}}$ (U76), $B$. diazoefficiens USDA $110^{\mathrm{T}}$ (U110), and B. japonicum USDA123 (U123). In half of the WT set microcosms (W), U110 was the USDA110 ${ }^{\mathrm{T}}$ wild-type strain possessing the nosZ gene, and in the other half of the Mut set (D), U110 was the USDA110 $\Delta$ nos $Z$ mutant that lacks the nos $Z$ gene. The other three strains remained the same in all incubations. Microcosm nomenclature follows the abbreviations above, such that ANW_H indicates a microcosm with andosol, non-flooding condition, WT set, incubated at high temperature. 
2A). In ADS microcosms with the Mut set, USDA110 $\Delta$ nos $Z$ remained at a low relative abundance in non-flooded and flooded microcosms. Strain USDA6 ${ }^{\mathrm{T}}$ remained in high abundance in non-flooded microcosms and slightly decreased in flooded microcosms though only significantly observed at higher incubation temperature. Meanwhile, USDA $76^{\mathrm{T}}$ slightly increased under higher temperatures regardless of the water status. In GLS microcosms with the WT set, changes in the community were as clearly detected as those in ADS microcosms (Fig. $2 B$ ). The relative abundance of USDA $110^{\mathrm{T}} \mathrm{wt}$ was initially high in flooded microcosms and remained so during the incubation period. Although strains $\mathrm{USDA}^{\mathrm{T}}$ and $\mathrm{USDA}^{7} 6^{\mathrm{T}}$ increased in flooded and non-flooded microcosms, their abundances were lower than those of USDA $110^{\mathrm{T}} \mathrm{wt}$ in flooded microcosms. The abundance of USDA $110^{\mathrm{T}} \mathrm{Wt}$ significantly decreased from an initially high level in non-flooded microcosms. On the other hand, the abundance of USDA110 $\Delta$ nos $Z$ significantly decreased in flooded and non-flooded microcosms (except at a high temperature in flooded microcosms). $\mathrm{USDA}^{\mathrm{T}}$ and USDA $76^{\mathrm{T}}$ in GLS soil both slightly increased in flooded and non-flooded microcosms due to the low survivability of the mutant, similar to non-flooded microcosms with the WT set. USDA123 also reached a relatively high abundance in non-flooded low temperature incubations.

In comparisons of community structures in the 30-d and 60-d incubations under the same treatment, a significant difference was not observed in most community structures (Fig. 2 and Table S2). Since changes in the community structures of these experimental conditions appeared to reach stable phases in the 30-d incubation and maintained similar communities under the experimental conditions employed in the present study, further multivariate analyses, a cluster analysis and MDS analysis, were conducted for all data as set data for comparisons among all treatments and between soil types.

\section{Cluster analysis}

The dendrogram based on the similarity indices of community structures in 30- and 60-d incubations indicated four major clusters: I, II, III, and IV (Fig. 3). Cluster numbers were decided from the ordering of the clusters in the MDS plots (Fig. 4). Based on strain abundance from Fig. 2, cluster I was comprised of communities in which USDA6 ${ }^{\mathrm{T}}$ and USDA76 ${ }^{\mathrm{T}}$ were dominant in microcosms with some WT sets in nonflooded and most Mut sets. Cluster I, having the lowest similarity index values, was strongly divided from the other clusters. Cluster IV was comprised of communities in which USDA $110^{\mathrm{T}} \mathrm{wt}$ was dominant in flooded microcosms. Cluster II was comprised of communities in which the dominant strains were predominantly USDA6 $6^{\mathrm{T}}$ and $\mathrm{USDA} 76^{\mathrm{T}}$ in nonflooded microcosms with $110^{\mathrm{T}} \mathrm{wt}$. Cluster III was mostly comprised of flooded microcosms and was closely related to cluster IV.

\section{MDS analysis of bradyrhizobial community structures}

MDS plots of the community structures of the different microcosms were classified into four clusters: I, II, III, and IV, based on the criteria that formed the clusters in the dendrogram (Fig. 4). Flooded microcosms containing the WT sets formed an apparently independent cluster from non-flooded microcosms and microcosms with the Mut sets, regardless of

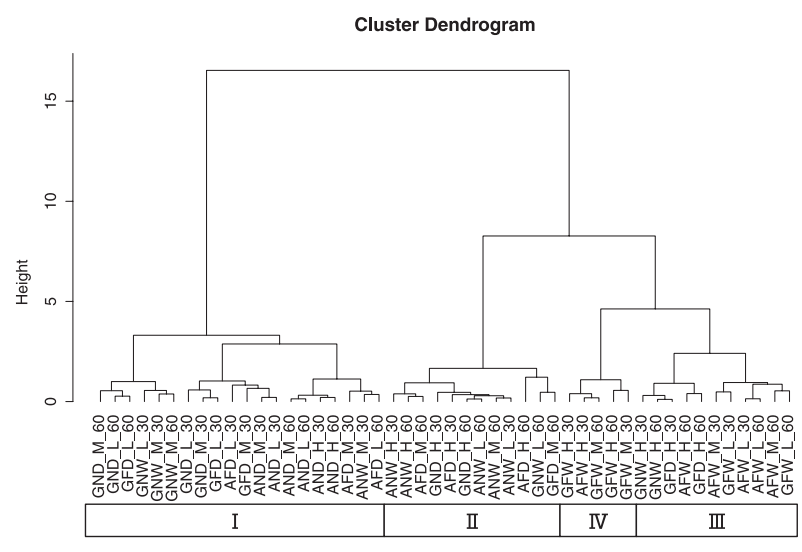

Fig. 3. Dendrogram of bradyrhizobial community structures of microcosms in 30- and 60-d incubations. The dendrogram was constructed by the Ward method using Bray-Curtis Indices as distances among communities.

Community nomenclature is a five-part name denoting soil type (A for ADS, G for GLS), the water status ( $\mathrm{N}$ for non-flooded, $\mathrm{F}$ for flooded), bradyrhizobial strain variant (W for a wild-type possessing the nos $Z$ gene and $\mathrm{D}$ for a mutant of the same strain that lacks the nos $Z$ gene), temperature $\left(\mathrm{H}\right.$ for $30^{\circ} \mathrm{C}, \mathrm{M}$ for $25^{\circ} \mathrm{C}$, and $\mathrm{L}$ for $20^{\circ} \mathrm{C}$ ), and incubation period ( 30 for $30 \mathrm{~d}$ and 60 for $60 \mathrm{~d}$ ); hence, ANW_H 30 indicates a microcosm with andosol, non-flooding conditions, the WT set, incubated at a high temperature for $30 \mathrm{~d}$.

the soil type (Fig. 5A and C). Microcosms with the Mut sets overlapped with flooded and non-flooded microcosms, regardless of the soil type (Fig. 5B and D). Community structures in flooded microcosms containing Mut set strains displayed a wider distribution in the MDS space than those with the nonflooded Mut set, with differentiation being more pronounced in flooded and high temperature incubations.

\section{PCA of bradyrhizobial community structures}

The results of PCA on bradyrhizobial community structures are shown in Fig. 6. A high cumulative proportion of variance was contained within the first two principal components (65\% on PC1 and $23 \%$ on PC2 for WT sets and $64 \%$ on PC1 and $26 \%$ on PC2 for Mut sets), and most of the relationships among the four strains under the experimental conditions used in this study were explained using 2D-PCA plots. Strains $\mathrm{USDA}^{\mathrm{T}}$ and USDA7 $6^{\mathrm{T}}$ became dominant under the aerobic conditions of non-flooded microcosms, whereas USDA $110^{\mathrm{T}} \mathrm{wt}$ became dominant under the reducing conditions of flooded treatments. As a result, a high proportion of dominance under the reducing conditions was observed with USDA $110^{\mathrm{T}} \mathrm{wt}$, which was detected in cluster IV. In contrast, since USDA110 $\Delta$ nos $Z$ showed decreased abundance in flooded microcosms, the proportion of variance observed with USDA110 $\operatorname{nos} Z$ was markedly lower than that of the WT strain. Most communities were detected in cluster I, whereas no communities belonging to cluster IV were detected. These results were supported by the results of MANOVA with high $\mathrm{F}$ values for the effect of flooded conditions in the WT set and relatively low values in the Mut set (Table 1). Additionally, USDA $76^{\mathrm{T}}$ increased its abundance at higher temperatures, whereas USDA123 slightly increased its abundance at lower temperatures (Fig. 2 and Table S2). Thus, a negative correlation was detected between strains USDA76 ${ }^{\mathrm{T}}$ and USDA123 on PC2 (Fig. 6A). Based on the results of 




Fig. 4. Two-dimensional-multidimensional-scaling (2D-MDS) plots of bradyrhizobial community structures of all microcosms in 0-, 30-, and 60-d incubations.

Community nomenclature is a five-part name denoting soil type (A for ADS, G for GLS), the water status (N for non-flooded, F for flooded), bradyrhizobial strain variant (W for a wild-type possessing the nos $Z$ gene and $\mathrm{D}$ for a mutant of the same strain that lacks the nos $Z$ gene), temperature ( $\mathrm{H}$ for $30^{\circ} \mathrm{C}, \mathrm{M}$ for $25^{\circ} \mathrm{C}$, and $\mathrm{L}$ for $20^{\circ} \mathrm{C}$ ), and incubation period ( 30 for $30 \mathrm{~d}$ and 60 for $60 \mathrm{~d}$ ). Clusters I, II, III, and IV correspond to those discriminated by the dendrogram in Fig. 3. The positions denoted by AW, AD, GW, and GD are MDS plots for the microcosms of different soil types (A or G) by bradyrhizobial strain variant (W or D) in a 0 -d incubation.

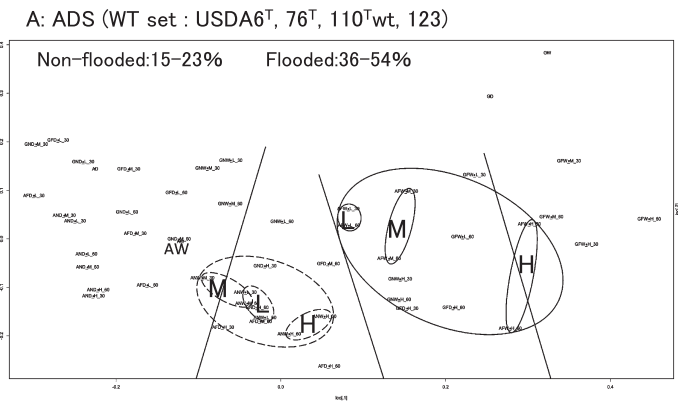

B: ADS (Mut set : USDA6 $6^{\top}, 76^{\top}, 110 \triangle$ nos $Z, 123$ )

C: GLS (WT set : USDA6 ${ }^{\top}, 76^{\top}, 110^{\top} w t, 123$ )
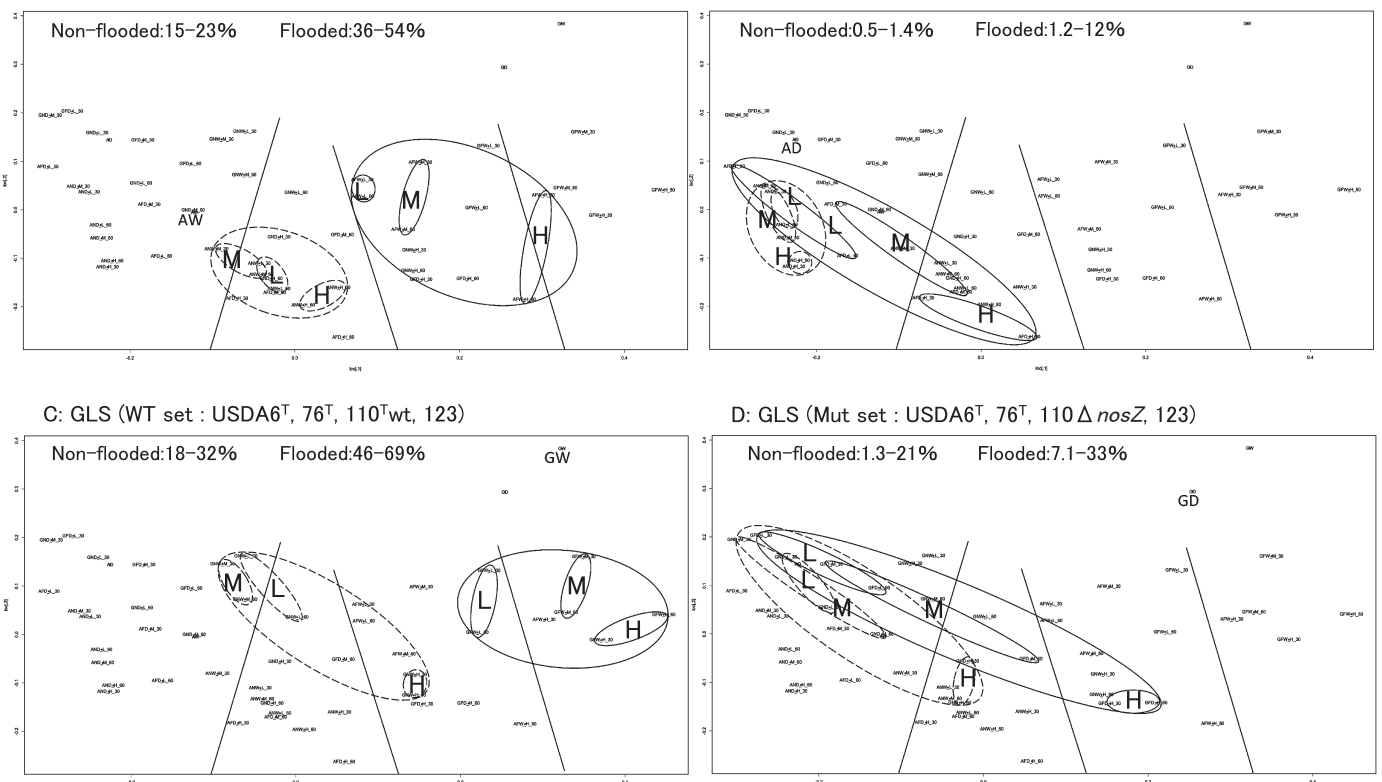

D: GLS (Mut set : USDA6 ${ }^{\top}, 76^{\top}, 110 \Delta \operatorname{nos} Z, 123$ )



Fig. 5. Differences in community structures between microcosms under flooding conditions (ellipses with solid lines) and non-flooding conditions (ellipses with dashed lines) within the 2D-MDS space.

The percentages of "flood" or "non-flood" refer to the range of abundance (\%) of the Bradyrhizobium diazoefficiens USDA110 strain in the 2D-MDS ellipse space for the respective microcosms being characterized. The ellipses denoted by H, M, or L indicate the characteristic 2D-MDS space for the respective subsets of microcosms of high, medium, and low incubation temperatures. The four panels are the analysis of the characteristic space for microcosms containing andosol (A and B) or gray lowland soil (C and D) and the WT set (A and C) or Mut set (B and D) containing variant strains of $B$. diazoefficiens within the bradyrhizobial community structure. 

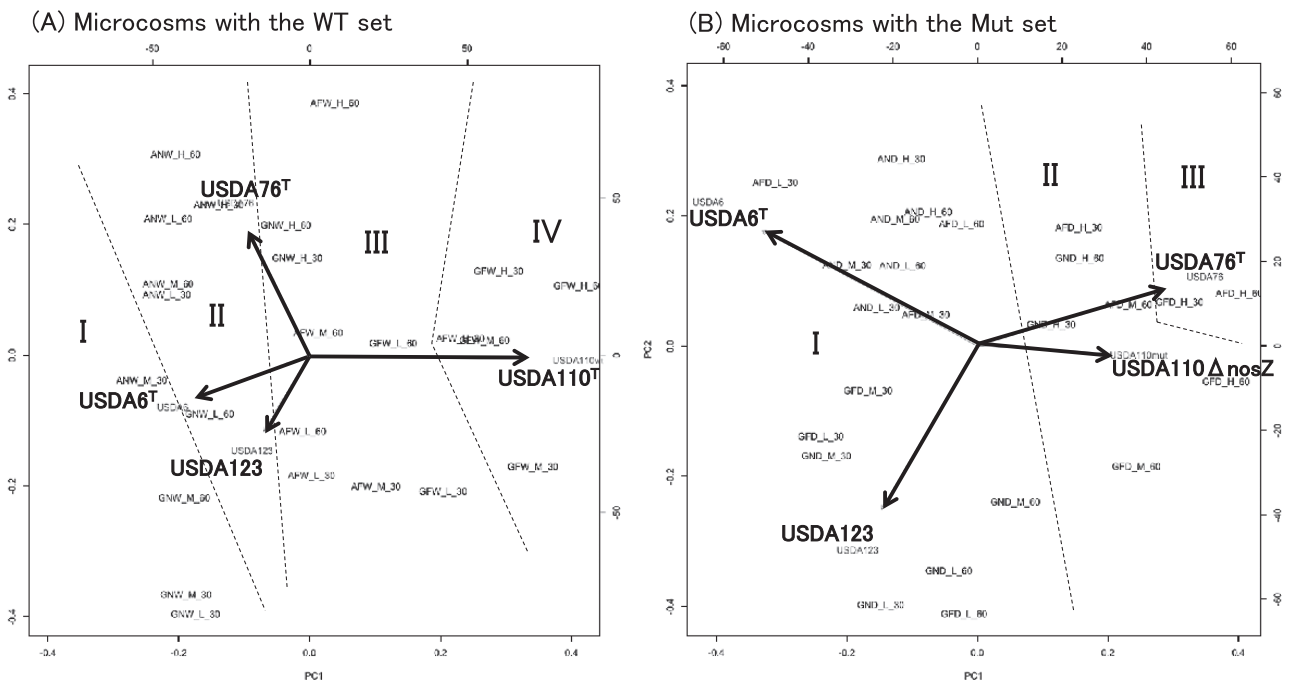

Fig. 6. Principal-component analysis of all community structures in 30- and 60-d incubations separated into microcosms according to whether the Bradyrhizobium diazoefficiens strain was USDA110 ${ }^{\mathrm{T}} \mathrm{wt}$ (A) or the USDA110 $\Delta$ nosZ mutant (B).

Numbers on PCA plots are cluster numbers based on the dendrogram from the cluster analysis. Arrows are the percentage contributions of each strain affecting the community structures.

Table 1. Results of a multivariate analysis of variance (Pillai's trace) on community structures in microcosms.

\begin{tabular}{lllll}
\hline & ADS WT set & ADS Mut set & GLS WT set & GLS Mut set \\
\hline Flood condition (F) & $F(1,34)=28.03, p=4.42 \mathrm{e}-09$ & $F(1,34)=9.09, p=0.000169$ & $F(1,34)=36.07, p=2.22 \mathrm{e}-10$ & $F(1,34)=4.50, p=0.00961$ \\
Temperature (T) & $F(2,33)=4.12, p=0.00148$ & $F(2,33)=3.40, p=0.00561$ & $F(2,33)=3.71, p=0.00319$ & $F(2,33)=5.01, p=0.000292$ \\
Incubation period (IP) & $F(1,34)=4.96, p=0.00614$ & $F(1,34)=2.71, p=0.0612$ & $F(1,34)=1.65, p=0.198$ & $F(2,34)=5.29, p=0.00445$ \\
$\mathrm{~F} \times \mathrm{T}$ & $F(5,30)=7.97, p=4.45 \mathrm{e}-11$ & $F(5,30)=5.11, p=3.49 \mathrm{e}-07$ & $F(5,30)=8.99, p=2.56 \mathrm{e}-12$ & $F(5,30)=3.03, p=0.000580$ \\
$\mathrm{~F} \times \mathrm{IP}$ & $F(3,32)=5.91, p=1.54 \mathrm{e}-06$ & $F(3,32)=3.64, p=0.000610$ & $F(3,32)=4.83, p=2.51 \mathrm{e}-05$ & $F(3,32)=3.10, p=0.00259$ \\
$\mathrm{~T} \times \mathrm{IP}$ & $F(5,30)=2.86, p=0.00106$ & $F(5,30)=2.32, p=0.00751$ & $F(5,30)=2.01, p=0.0224$ & $F(5,30)=3.24, p=0.000271$ \\
$\mathrm{~F} \times \mathrm{T} \times \mathrm{IP}$ & $F(11,24)=6.30, p=3.94 \mathrm{e}-11$ & $F(11,24)=3.58, p=3.23 \mathrm{e}-06$ & $F(11,24)=5.20, p=2.70 \mathrm{e}-09$ & $F(11,24)=2.18, p=0.00300$ \\
\hline
\end{tabular}

MANOVA, the effects of temperature on community structures were weaker than those of flooded conditions in this study (Table 1).

\section{Discussion}

In the present study, we attempted to demonstrate the effects of strains possessing the nos $Z$ gene on bradyrhizobia community structures in microcosm soils under different temperatures and oxidation-reduction conditions.

Community structures in flooded and non-flooded microcosms with the WT set, containing strains $\mathrm{USDA}^{\mathrm{T}}, 76^{\mathrm{T}}$, $110^{\mathrm{T}} \mathrm{wt}$, and 123 , displayed shifts for similar community structures in ADS and GLS soil types. Changes in community structures in WT set microcosms that were flooded were more pronounced than in those that were not flooded. USDA $110^{\mathrm{T}} \mathrm{wt}$, which is nos $Z$ positive (nos $Z+$ ), became dominant under the anaerobic conditions in flooded microcosms (Fig. 2). In flooded microcosms, the dominance of USDA $110^{\mathrm{T}}$ wt was more pronounced in GLS than in ADS microcosms. In Mut set microcosms, USDA110 $\Delta$ nosZ, lacking $\operatorname{nos} Z(\Delta$ nos $Z$ ), did not become dominant in any of the flooded microcosms, and community structures with this $\Delta n o s Z$ mutant displayed similar changes under flooded and nonflooded conditions. Although dissolved oxygen concentrations were not significantly different among flooded microcosms, ORP values decreased more rapidly in GLS than in ADS, and significantly decreased before $14 \mathrm{~d}$ of incubation (Fig. 1).
Although the reason for the difference in ORP changes between soil types currently remains unclear, a previous study reported that the difference in ORP changes was affected by soil types, and soil that developed from silt showed rapid decreases in ORP under flooded conditions (42). Relationships among nitrate, $\mathrm{pH}$, and $\mathrm{EC}$ have been reported by many researchers. Positive correlations have been reported between nitrate concentrations and EC values, and negative correlations between $\mathrm{pH}$ and nitrate indicate (21). Based on EC and $\mathrm{pH}$ values, denitrification may have occurred during the incubation period. Denitrification in soil is expressed as ORP values in the range of approximately $\pm 100 \mathrm{mV}$, and the optimal range of values to promote denitrification is $\pm 50 \mathrm{mV}$ (44). Therefore, denitrification by bradyrhizobia in flooded GLS microcosms may have occurred and reached a peak after approximately $14 \mathrm{~d}$ of incubation, and denitrification activities may have decreased until approximately $30 \mathrm{~d}$ of incubation. In flooded ADS microcosms, denitrification by bradyrhizobia may have occurred later than in GLS microcosms and may then have been expressed for most of the incubation period because ORP values remained in the optimal range for denitrification. These results suggest that the ORP and DOC values reached were in a range that led to changes and differentiation in bradyrhizobial community structures in different soil types. We previously indicated that temperature is one of the factors affecting the construction of bradyrhizobial community structures in soils in a microcosm study (24). The present 
results also suggest that soil ORP is an environmental factor affecting the ecology and occupancy of bradyrhizobia in soils.

The cluster analysis and MDS analysis indicated that all microcosms in cluster IV were flooded and contained USDA $110^{\mathrm{T}} \mathrm{wt}$ as the dominant strain (Fig. 2, 3, and 4). Furthermore, cluster I mostly comprised microcosms with USDA110 $\operatorname{nos} Z$ (AND, AFD, GND, and GFD) and GNW with USDA1 $10^{\mathrm{T}} \mathrm{wt}$ (Fig. 3), and was relatively well separated from clusters mostly comprised of flooded microcosms containing USDA $110^{\mathrm{T}} \mathrm{wt}$ (clusters III and IV). As shown in Fig. 5 , the positions of the different community structure clusters were similar between ADS and GLS microcosms, with the greatest influence shown by flooding and USDA1 $10^{\mathrm{T}}$ deployed. The slight difference observed in the positions of the different community structures between ADS and GLS microcosms may be due to different ORP profiles in the two soils. In microcosms with the Mut set, differences in changes in community structures were not readily distinguishable between flooded and non-flooded microcosms, particularly for the lower temperature incubations (Fig. 5B and D). However, higher temperatures had a stronger influence on the separation of community structures in microcosms with the Mut set under flooded conditions than under lower temperatures and non-flooded conditions. This result suggests that other functions that were influenced by temperature, except for nos $Z$, function in the differentiation of community structures under flooded conditions. Sameshima-Saito et al. (29) reported that symbiotic USDA $110^{\mathrm{T}}$, indicating the final step in denitrification activity from $\mathrm{N}_{2} \mathrm{O}$ to $\mathrm{N}_{2}$, reduces $\mathrm{N}_{2} \mathrm{O}$ surrounding the soybean root system. Additionally, the present results suggest that the ability to undertake the final step in denitrification activity is an advantage to strains that possess the nos $Z$ gene, and these strains will outperform strains in flooded soil that do not possess this gene, thereby altering the bradyrhizobial community structure. According to the study by Thauer et al. (40), total energy acquisition by complete denitrification from nitrate to $\mathrm{N}_{2}$ gas is $1120.9 \mathrm{~kJ}$, and partial energy acquisition from $\mathrm{N}_{2} \mathrm{O}$ to $\mathrm{N}_{2}$ is $341.4 \mathrm{~kJ}$. Energy acquisition by complete denitrification activity containing $\mathrm{N}_{2} \mathrm{O}$ reductase is higher by approximately $30 \%$ than that of incomplete denitrification until $\mathrm{N}_{2} \mathrm{O}$. This difference in energy acquisition by denitrification may be a competitive advantage to bradyrhizobia possessing the nos $Z$ gene in flooded soils. Differences between soil types in the community structure response to anaerobic conditions may be attributable to differences in changes in ORP values after flooding. Temperature is also an important factor in the establishment of bradyrhizobial community structures, and will always need to be considered when verifying the effects of the expression of denitrificationrelated genes on community structures. The PCA of bradyrhizobial community structures showed a strong negative correlation between strains USDA6 ${ }^{\mathrm{T}}$ and USDA $110^{\mathrm{T}} \mathrm{wt}$ on PC1 (Fig. 6A). Although this negative correlation was also detected between USDA $6^{\mathrm{T}}$ and USDA110 $\Delta$ nos $Z$, the relative contribution of USDA $110 \Delta$ nos $Z$ to total variance was markedly less than that of USDA $110^{\mathrm{T}} \mathrm{wt}$ (Fig. 6B). These results suggest that a negative correlation may exist due to the interaction between strains USDA $110^{\mathrm{T}}($ nos $Z+)$ and USDA6 ${ }^{\mathrm{T}}$ (nosZ-). Shiina et al. (33) reported that nos Z- strains were dominant in andosols and nos $Z+$ strains were dominant in alluvial soils in Japan. Our previous study (26) also detected a high abundance of strains belonging to the USDA $110^{\mathrm{T}}$ cluster in gray lowland and gley soils in Japan, indicating that flooding and poor drainage are important environmental factors favoring bradyrhizobia strains possessing the nos $Z$ gene. These results were supported by those of MANOVA (Table 1). The effects of flooded conditions on the bradyrhizobial community structures of WT sets were stronger than those of other environmental factors in the present study. On the other hand, the effects of flooded conditions on the community structures of the Mut sets were weaker than those of the WT sets. Furthermore, GLS microcosms may be affected more by flooded conditions than ADS microcosms due to differences in the ORP status. Additionally, there was a negative correlation between strains USDA76 ${ }^{\mathrm{T}}$ and USDA123 on PC2 (Fig. 6A). This negative correlation may have been induced by temperature during the incubation. USDA $76^{\mathrm{T}}$ was slightly more dominant at higher temperatures, and USDA123 at lower temperatures. This is in accordance with the known geographical distribution of bradyrhizobia strains in Japan and the United States $(26,27,35)$. Furthermore, many studies have suggested that the relationship between genomic diversity and the field distribution of indigenous soybean-nodulating rhizobia is affected by soil environmental properties such as temperature being influenced by latitude and altitude, phosphorus content, EC, and soil pH $(1,18,22,34,36,43)$. The present results also indicate that the mechanism of dominance in poorly aerated soils under field conditions is related to the denitrification activity of bradyrhizobia. Since ORP values less than $-200 \mathrm{mV}$ are indicative of completely anaerobic conditions that induce methane fermentation, paddy fields with ORP values in the range of $\pm 100 \mathrm{mV}$, and particularly in the range of $\pm 50 \mathrm{mV}$ (44), present optimum field conditions for the dominance of bradyrhizobia possessing denitrification activity, such as nos $Z+$ bradyrhizobial strains. Bradyrhizobia that have incomplete denitrification will become slightly more dominant in aerobic soils, even those in fields converted from paddy to upland cropping for an extended period. The results of MANOVA suggest that the change in ORP caused by soil saturation has a greater effect on bradyrhizobial community structures than temperature changes.

In the present study, we demonstrated that the soil water status is one of the important environmental factors affecting soil bradyrhizobial community structures. The ORP status appears to be an environmental factor that has a greater impact on bradyrhizobial community structures than temperature, at least in the range of the temperatures examined in the present study. Investigations on bradyrhizobial community structures not only under a wider range of temperatures, but also with a wide range of various environmental factors are needed in order to elucidate rhizobial ecological traits. Furthermore, other microorganisms may be concerned with the establishment of bradyrhizobial community structures. Further studies are needed in order to elucidate rhizobial ecology including other soil microorganisms. B. diazoefficiens USDA $110^{\mathrm{T}}$ has not only a strong nitrogen fixation ability, but also the ability to undertake complete denitrification (28). Strains with these abilities may make an important contribution to sustainable soybean production (13). Our results suggest that bradyrhizobial community structures are manipulated to favor or maintain 
useful strains including $B$. diazoefficiens USDA $110^{\mathrm{T}}$ through soil management that involves periodic water flooding, such as the soybean-paddy rotation system. Further investigations on the ecological functions of bradyrhizobial communities that lead to control by agricultural management are important for the continued development of sustainable soybean production.

\section{Acknowledgements}

This study was supported by a grant from the Institute for Fermentation, Osaka, Japan, and by the JSPS KAKENHI (Grant-inAid for Scientific Research (B) no. 26310313) from the Japanese Ministry of Education, Culture, Sports, Science and Technology (MEXT) to Y. S. This work was also supported in part by the JSPS KAKENHI (Grant-in-Aid for Scientific Research (A) no. 26252065) from MEXT and a grant from the Ministry of Agriculture, Forestry, and Fisheries of Japan (BRAIN) to K. M.

\section{References}

1. Adhikari, D., M. Kaneto, K. Itoh, K. Suyama, B.B. Pokharel, and Y.K. Gaihre. 2012. Genetic diversity of soybean-nodulating rhizobia in Nepal in relation to climate and soil properties. Plant Soil 357:131145 .

2. Bedmar, E.J., E.F. Robles, and M.J. Delgado. 2005. The complete denitrification pathway of the symbiotic, nitrogen-fixing bacterium Bradyrhizobium japonicum. Biochem. Soc. Trans. 33:141-144.

3. Bray, J.R., and J.T. Curtis. 1957. An ordination of the upland forest communities of southern Wisconsin. Ecol. Monogr. 27:325-349.

4. Chen, W.X., G.H. Yan, and J.L. Li. 1988. Numerical taxonomic study of fast-growing soybean rhizobia and a proposal that Rhizobium fredii be assigned to Sinorhizobium gen. nov. Int. J. Syst. Bacteriol. 38:392-397.

5. Cultivated Soil Classification Committee. 1996. Classification of Cultivated Soils in Japan, Third approximation. National Institute of Agro-Environmental Science, Tsukuba, Japan.

6. Delamuta, J.R.M., R.A. Ribeiro, E. Ormeño-Orrillo, I.S. Melo, E. Martínez-Romero, and M. Hungria. 2013. Polyphasic evidence supporting the reclassification of Bradyrhizobium japonicum group Ia strains as Bradyrhizobium diazoefficiens sp. nov. Int. J. Sys. Evol. Microbiol. 63:3342-3351.

7. Faith, D.P., P.R. Minchin, and L. Belbin. 1987. Compositional dissimilarity as a robust measure of ecological distance. Vegetatio. 69:57-68.

8. Hirayama, J., S. Eda, H. Mitsui, and K. Minamisawa. 2011. Nitratedependent $\mathrm{N}_{2} \mathrm{O}$ emission from intact soybean nodules via denitrification by Bradyrhizobium japonicum bacteroids. Appl. Environ. Microbiol. 77:8787-8790.

9. Hungria, M., L.H. Boddey, M.A. Santos, and M.A.T. Vargas. 1998. Nitrogen fixation capacity and nodule occupancy by Bradyrhizobium japonicum and B. elkanii strains. Biol. Fertil. Soils 27:393-399.

10. Inaba, S., K. Tanabe, S. Eda, S. Ikeda, A. Higashitani, H. Mitsui, and K. Minamisawa. 2009. Nitrous oxide emission and microbial community in the rhizosphere of nodulated soybeans during the late growth period. Microbes Environ. 24:64-67.

11. Inaba, S., F. Ikenishi, M. Itakura, M. Kikuchi, S. Eda, N. Chiba, C. Katsuyama, Y. Suwa, H. Mitsui, and K. Minamisawa. 2012. $\mathrm{N}_{2} \mathrm{O}$ emission from degraded soybean nodules depends on denitrification by Bradyrhizobium japonicum and other microbes in the rhizosphere. Microbes Environ. 27:470-476.

12. IPCC. 2007. Climate change 2007: The physical science basis. Contribution of working group I to the fourth assessment report of the Intergovernmental Panel on Climate Change, p 996. In. S. Solomon, D. Qin, M. Manning, Z. Chen, M. Marquis, K.B. Averyt, M. Tignor, and H.L. Miller (ed.), Cambridge University Press, Cambridge, United Kingdom and New York, NY,

13. Itakura, M., Y. Uchida, H. Akiyama, et al. 2013. Mitigation of nitrous oxide emissions from soils by Bradyrhizobium japonicum inoculation. Nature Climate Change 3:208-212.

14. Jarvis, B.D.W., H.L. Downer, and J.P.W. Young. 1992. Phylogeny of fast-growing soybean-nodulating rhizobia supports synonymy of Sinorhizobium and Rhizobium and assignment to Rhizobium fredii. Int. J. Syst. Bacteriol. 42:93-96.
15. Jordan, D.C. 1982. Transfer of Rhizobium japonicum Buchanan 1980 to Bradyrhizobium gen. nov., a genus of slow-growing, root nodule bacteria from leguminous plants. Int. J. Syst. Bacteriol. 32:136-139.

16. Keyser, H.H., and F. Li. 1992. Potential for increasing biological nitrogen fixation in soybean. Plant Soil 141:119-135.

17. Kuykendall, L.D., B. Saxena, T.E. Devine, and S.E. Udell. 1992. Genetic diversity in Bradyrhizobium japonicum Jordan 1982 and a proposal for Bradyrhizobium elkanii sp. nov. Can. J. Microbiol. 38:501-505.

18. Li, Q.Q., E.T. Wang, Y.Z. Zhang, Y.M. Zhang, C.F. Tian, X.H. Sui, W.F. Chen, and W.X. Chen. 2011. Diversity and biogeography of rhizobia isolated from root nodules of Glycine max grown in Hebei province, China. Microb. Ecol. 61:917-931.

19. Michie, M.G. 1982. Use of the Bray-Curtis similarity measure in cluster analysis of foraminiferal data. Math. Geol. 14:661- 667.

20. Minamisawa, K., Y. Nakatsuka, and T. Isawa. 1999. Diversity and field site variation of indigenous populations of soybean bradyrhizobia in Japan by fingerprints with repeated sequences RS $\alpha$ and RS $\beta$. FEMS Microbiol. Ecol. 29:171-178.

21. Patriquin, D.G., H. Blaikie, M.J. Patriquin, and C. Yang. 1993. On-farm measurements of $\mathrm{pH}$, electrical conductivity and nitrate in soil extracts for monitoring coupling and decoupling of nutrient cycles. Biol. Agri. Hort. 9:231-272.

22. Risal, C.P., T. Yokoyama, N. Ohkama-Ohtsu, S. Djedidi, and H. Sekimoto. 2010. Genetic diversity of native soybean bradyrhizobia from different topographical regions along the southern slopes of the Himalayan mountains in Nepal. Syst. Appl. Microbiol. 33:416-425.

23. Saeki, Y., N. Aimi, S. Tsukamoto, T. Yamakawa, Y. Nagatomo, and S. Akao. 2006. Diversity and geographical distribution of indigenous soybean-nodulating bradyrhizobia in Japan. Soil Sci. Plant Nutr. 52:418-426.

24. Saeki, Y., S. Ozumi, A. Yamamoto, Y. Umehara, M. Hayashi, and G.C. Sigua. 2010. Changes in population occupancy of bradyrhizobia under different temperature regimes. Microbes Environ. 25:309-312.

25. Saeki, Y. 2011. Characterization of soybean-nodulating rhizobial communities and diversity, p. 163-184. In S Aleksandra (ed.), Soybean-Molecular Aspects of Breeding. Intech, Rijeka.

26. Saeki, Y., S. Shiro, T. Tajima, A. Yamamoto, R. Sameshima-Saito, T. Sato, and T. Yamakawa. 2013. Mathematical ecology analysis of geographical distribution of soybean-nodulating bradyrhizobia in Japan. Microbes Environ. 28:470-478.

27. Saeki, Y., and S. Shiro. 2014. Comparison of soybean-nodulating bradyrhizobia community structures along north latitude between Japan and USA, p. 195-223. In T. Ohyama (ed.), Advances in Biology and Ecology of Nitrogen Fixation. Intech, Rijeka.

28. Sameshima-Saito, R., K. Chiba, and K. Minamisawa. 2006. Correlation of denitrifying capability with the existence of nap, nir, nor and nos genes in diverse strains of soybean bradyrhizobia. Microbes Environ. 21:174-184.

29. Sameshima-Saito, R., K. Chiba, J. Hirayama, M. Itakura, H. Mitsui, S. Eda, and K. Minamisawa. 2006. Symbiotic Bradyrhizobium japonicum reduces $\mathrm{N}_{2} \mathrm{O}$ surrounding the soybean root system via nitrous oxide reductase. Appl. Environ. Microbiol. 72:2526-2532.

30. Sawada, Y., K. Miyashita, I. Tanabe, and K. Kato. 1989. Hup phenotype and serogroup identity of soybean nodulating bacteria isolated from Japanese soil. Soil Sci. Plant Nutr. 35:281-288.

31. Scholla, M.H., and G.H. Elkan. 1984. Rhizobium fredii sp. nov., a fast-growing species that effectively nodulates soybeans. Int. J. Syst. Bacteriol. 34:484-486.

32. Senaratne, R., C. Amornpimol, and G. Hardarson. 1987. Effect of combined nitrogen on nitrogen fixation of soybean (Glycine max L. Merrill.) as affected by cultivar and rhizobial strain. Plant Soil 103:45-50.

33. Shiina, Y., M. Itakura, H. Choi, Y. Saeki, M. Hayatsu, and K. Minamisawa. 2014. Relationship between soil type and $\mathrm{N}_{2} \mathrm{O}$ reductase genotype (nosZ) of indigenous soybean bradyrhizobia: nosZ-minus populations are dominant in andosols. Microbes Environ. 29:420-426.

34. Shiro, S., A. Yamamoto, Y. Umehara, M. Hayashi, N. Yoshida, A. Nishiwaki, T. Yamakawa, and Y. Saeki. 2012. Effect of Rj genotype and cultivation temperature on the community structure of soybeannodulating bradyrhizobia. Appl. Environ. Microbiol. 78:1243-1250.

35. Shiro, S., S. Matsuura, R. Saiki, G.C. Sigua, A. Yamamoto, Y. Umehara, M. Hayashi, and Y. Saeki. 2013. Genetic diversity and geographical distribution of indigenous soybean-nodulating bradyrhizobia in the United States. Appl. Environ. Microbiol. 79:3610-3618. 
36. Shiro, S., C. Kuranaga, A. Yamamoto, R. Sameshima-Saito, and Y. Saeki. 2016. Temperature-dependent expression of nodC and community structure of soybean-nodulating bradyrhizobia. Microbes Environ. 31:27-32.

37. Siqueira, A.F., E. Ormeño-Orrillo, R.C. Souza, et al. 2014. Comparative genomics of Bradyrhizobium japonicum CPAC 15 and Bradyrhizobium diazoefficiens CPAC 7: elite model strains for understanding symbiotic performance with soybean. BMC Genomics $15: 420-439$.

38. Sugawara, M., and M.J. Sadowsky. 2013. Influence of elevated atmospheric carbon dioxide on transcriptional responses of Bradyrhizobium japonicum in the soybean rhizoplane. Microbes Environ. 28:217-227.

39. Suzuki, K., H. Oguro, T. Yamakawa, A. Yamamoto, S. Akao, and Y. Saeki. 2008. Diversity and distribution of indigenous soybeannodulating rhizobia in the Okinawa Islands, Japan. Soil Sci. Plant Nutr. 54:237-246.

40. Thauer, R.K., K. Jungermann, and K. Decker. 1977. Energy conservation in chemotrophic anaerobic bacteria. Bacteriol. Rev. 41:100-180.
41. Vincent, J.M. 1970. A Manual for the Practical Study of the Rootnodule Bacteria. International Biological Program. Blackwell Scientific Publications, Oxford.

42. Wlodarczyk, T., P. Szarlip, M. Brzezinska, and U. Kotowska. 2007. Redox potential, nitrate content and $\mathrm{pH}$ in flooded Eutric Cambisol during nitrate reduction. Res. Agr. Eng. 53:20-28.

43. Yokoyama, T. 2005. Effects of temperature on competition for nodulation in phylogenetically different Bradyrhizobium strains. Jpn. J. Soil Sci. Plant Nutr. 76:599-607. (In Japanese)

44. Yoon, S-H. 2016. Membrane bioreactor processes, p. 164-166. CRC Press, Taylor \& Francis Group, LLC. FL.

45. Young, J.M. 2003. The genus name Ensifer Casida 1982 takes priority over Sinorhizobium Chen et al. 1988, and Sinorhizobium morelense Wang et al. 2002 is a later synonym of Ensifer adhaerens Casida 1982. Is the combination 'Sinorhizobium adhaerens' (Casida 1982) Willems et al. 2003 legitimate? Request for an opinion. Int. J. Syst. Evol. Microbiol. 53:2107-2110. 